\title{
Neoclassical Toroidal Plasma Viscosity Torque in Collisionless Regimes in Tokamaks
}

\author{
Y. Sun, ${ }^{1, *}$ Y. Liang, ${ }^{1}$ K. C. Shaing,,${ }^{2,3}$ H. R. Koslowski, ${ }^{1}$ C. Wiegmann,,${ }^{1}$ and T. Zhang ${ }^{1}$ \\ ${ }^{1}$ Institute for Energy Research-Plasma Physics, Forschungszentrum Jülich, Association EURATOM-FZJ, \\ Trilateral Euregio Cluster, 52425 Jülich, Germany \\ ${ }^{2}$ Institute for Space, Astrophysical and Plasma Sciences, National Cheng Kung Unversity, \\ Tainan, Taiwan 70101, Republic of China \\ ${ }^{3}$ Engineering Physics Department, University of Wisconsin, Madison, Wisconsin 53706, USA
}

(Received 28 July 2010; published 1 October 2010)

\begin{abstract}
Bumpiness in a magnetic field enhances the magnitude of the plasma viscosity and increases the rate of the plasma flow damping. A general solution of the neoclassical toroidal plasma viscosity (NTV) torque induced by nonaxisymmetric magnetic perturbation (NAMP) in the collisionless regimes in tokamaks is obtained in this Letter. The plasma angular momentum can be strongly changed, when there is a small deviation of the toroidal symmetry caused by a NAMP of the order of $0.1 \%$ of the toroidal field strength.
\end{abstract}

DOI: 10.1103/PhysRevLett.105.145002

PACS numbers: 52.55.Fa, 52.25.Fi, 52.55.Tn

It is known that the magnetic fields used in confining plasmas are usually spatially nonuniform or bumpy. The bumpiness of the fields increases the plasma viscosity and consequently the rate of the plasma flow damping. When the collision frequency is smaller than the bounce or transit frequency of the particles that traverse through the bumpiness of the fields, particles experience the resistance of the field when they either reflect back or slow down by the fields. This resistance enhances the plasma viscosity. The underlying physics can occur in any magnetized plasmas confined by the spatially nonuniform fields.

The magnetic field of a tokamak is designed to be toroidally symmetric. Realistically, there is always a slight nonaxisymmetric magnetic perturbation (NAMP) due to an intrinsic error field, magnetohydrodynamics (MHD) perturbations in the plasma and external magnetic perturbation applied to control edge localized modes (ELMs) [1,2] and resistive wall modes (RWMs) [3]. In stellarators, the plasma diffusion in collisionless regimes induced by the helical magnetic field has been developed since the 1960s [4]. The neoclassical viscosity can also be obtained by solving the drift kinetic equation numerically [5]. However, the ordering of the NAMP in tokamaks is different from that in stellarators. In the last few years, the neoclassical toroidal plasma viscosity (NTV) theory in different asymptotic limits of the collisionless regimes [6-11] has been developed to describe the plasma momentum dissipation induced by the NAMP field in tokamaks.

The importance of the NTV torque in momentum confinement has been highlighted by the most recent experimental observations. Strong magnetic braking effect without mode locking during the application of NAMP has been observed in the experiments in tokamaks [12-15]. The NTV torque is a good candidate to explain the observed braking effect.

The experimental regime in present tokamaks as well as the International Thermonuclear Experimental Reactor
(ITER) [16] covers both the $1 / \nu$ and $\nu-\sqrt{\nu}$ regimes and their transitions. Here, $\nu$ is the collisionality. The typical collisionality regime on DIII-D [13] and JET [15] are close to the transition of $1 / \nu$ and $\nu-\sqrt{\nu}$ regimes. Furthermore, particles with different energy are in different collisionality regimes. In order to model the toroidal plasma rotation with NAMP and compare it with the observation, we need to know the exact NTV solution in the transition regimes, as well as in the asymptotic limits of these collisionless regimes. However, there was no analytic solution in the transition regimes yet.

Recently, one approximate analytic general expression has been obtained by smoothly connecting the formula in these different regimes by Shaing et al. [17]. Another general analytic result has been obtained by using the simple Krook collisional operator by Park et al. [18]. However, this cannot include the boundary layer physics [7].

In this Letter, one general solution of NTV torque in these collisionless regimes in tokamaks is obtained by numerically solving the linearized bounce-averaged drift kinetic equation with pitch angle scattering collisional operator. The NTV in the transition regimes can be modeled with this method without additional approximations.

The magnetic field strength with NAMP can be written as

$$
B=B_{0}\left[1-\epsilon \cos \theta-\sum_{n} b_{n}(\theta) e^{\mathrm{in} \alpha}\right]
$$

where $B_{0}$ is the magnetic field strength on the magnetic axis, $\epsilon \approx r / R$ is the amplitude of the $\cos \theta$ component of the equilibrium field caused by toroidicity, $r$ and $R$ are the minor and major radius, respectively, $\alpha=q \theta-\zeta$ is the drift angle, $b_{n}=\sum_{m} b_{m n} e^{i(m-n q) \theta}$ is the $n$th Fourier harmonic of the perturbation field, and $b_{m n}$ are the Fourier coefficients of normalized perturbation field in $(\theta, \zeta)$ coordinates on the distorted flux surfaces [6]. The equilibrium magnetic field can be expressed as $\vec{B}=\psi_{p}^{\prime} \nabla \hat{V} \times \nabla \alpha$, 
where $2 \pi \psi_{p}$ is the poloidal magnetic flux, $\hat{V} \equiv V /\left(4 \pi^{2}\right), V$ is the plasma volume enclosed by the flux surface, and the prime denotes the derivative to $\hat{V}$. $(\hat{V}, \theta, \zeta)$ are the Hamada coordinates with Jacobin $J=1$.

The typical normalized collisionality $\nu_{*} \equiv \nu /\left(\epsilon^{3 / 2} \omega_{t}\right)$, satisfies $\left(\frac{\delta B / B}{\epsilon}\right)^{3 / 2} \ll \nu_{*} \ll 1$ in most tokamak operational regimes for present tokamaks as well as ITER. Here $\omega_{t}=$ $v_{t} / q R_{0}$ is the transient frequency. The collisionless detrapping or retrapping regime [8] and superbanana regime [10] are not usually accessible. The bounce-averaged drift kinetic equation can be applied for describing the NTV in the collisionless regimes in tokamaks.

The linearized bounce-averaged drift kinetic equation $[4,6,19]$ can be written as

$$
\omega_{d \alpha} \partial_{\alpha} f_{1}+\omega_{d \hat{V}} \partial_{\hat{V}} f_{M}=\frac{\nu_{d}}{2 \epsilon}\left\langle L\left(f_{1}\right)\right\rangle_{b},
$$

where $f_{1}$ is the perturbed distribution function, $\omega_{d \alpha}$ and $\omega_{d \hat{V}}$ are the bounce-averaged drift frequencies in the $\alpha$ and $\hat{V}$ direction, respectively, $f_{M}$ is the local Maxwellian distribution function, $\nu_{d}$ is the deflection frequency, $\langle L\rangle_{b} \approx$ $\partial_{\kappa^{2}}+F \partial_{\kappa^{2}}^{2}$ is the bounce-averaged pitch angle scattering collisional operator, $F \equiv 2\left[E / K-1+\kappa^{2}\right]$, and $\langle\ldots\rangle_{b}$ denotes the bounce average. $K\left(\kappa^{2}\right)$ and $E\left(\kappa^{2}\right)$ are the elliptic integrals of the first and second kind, respectively. For trapped particles, the pitch angle $\kappa^{2} \in[0,1] . \omega_{d \alpha}=$ $-q \omega_{E}-\omega_{B}$, in which $q \omega_{E}$ is the $\vec{E} \times \vec{B}$ drift frequency and $\omega_{B} \approx \omega_{B 0}[2 E / K-1] x$ is the magnetic drift frequency. $\omega_{B 0}$ is the magnetic drift of the deeply trapped particles with $x=1$ and $x=v^{2} / v_{t}^{2}$ is the normalized energy.

Without considering the coupling of different harmonics of the perturbed radial drift in the superbanana orbits [10], the magnetic flux surface averaged particle flux, $\Gamma=$ $\left\langle\int d \vec{v}\left(\vec{v}_{d} \cdot \nabla \hat{V}\right) f\right\rangle_{\psi}$, from different harmonics of the perturbation field can be calculated separately. Here $\langle A\rangle_{\psi} \equiv$ $\frac{1}{4 \pi^{2}} \oint d \theta \oint d \zeta A$ is the flux surface average of $A$, and $f$ is the distribution function of the particles.

By changing the flux into quadratic form [6], the general form of the $\Gamma$ for the $j$ th $(j=$ ions, electron) kind of particles can be written as

$$
\begin{gathered}
\Gamma_{j}=\rho_{j} R_{0}^{2} \frac{\sqrt{\epsilon} q^{2} \omega_{t j}^{2}}{2 \sqrt{2} \pi^{3 / 2} e_{j} \psi_{p}^{\prime}} \sum_{n} \lambda_{1, n}\left(V^{\zeta}-\omega_{n c, n}^{j}\right), \\
\omega_{n c, n}^{j} \equiv q\left(V^{\theta}+\omega_{*, j}-\omega_{*, i}+\frac{\lambda_{2, n}}{\lambda_{1, n}} \omega_{* T, j}\right), \\
\lambda_{j, n} \equiv \frac{1}{2} \int_{0}^{\infty} I_{\kappa n}(x)(x-5 / 2)^{j-1} x^{5 / 2} e^{-x} d x, \\
I_{\kappa n} \equiv \frac{\left|n\left\langle b_{n}\right\rangle_{b}\right|_{\kappa^{2}=0}^{2}}{\nu_{d} /(2 \epsilon)} \int_{0}^{1} 4 K F\left(I_{1}\left|\partial_{\kappa^{2}} f_{1 n}\right|\right)^{2} d \kappa^{2},
\end{gathered}
$$

where $\rho_{j}$ and $e_{j}$ are the mass density and electric charge of the $j$ th kind of particles, respectively, $V^{\zeta} \equiv \vec{V} \cdot \nabla \zeta$ and
$V^{\theta} \equiv \vec{V} \cdot \nabla \theta$ are the contravariants of ion flow. $\omega_{n c}$ is the general form of the so-called neoclassical "offset" rotation $[13,20]$. The diamagnetic frequencies $\omega_{*} \equiv \frac{1}{N e q} \frac{d}{d \psi_{p}} P$, and $\omega_{* T} \equiv \frac{1}{e q} \frac{d}{d \psi_{p}} T . I_{1}=\frac{\nu_{d} /(2 \epsilon)}{I_{0}}$ and $I_{0}=$ $\sqrt{\left[\nu_{d} /(2 \epsilon)\right]^{2}+\max \left[\left(n \omega_{d \alpha}\right)^{2}\right]}$. The perturbed distribution function is expanded as $f_{1}\left(\hat{V}, \alpha, \kappa^{2}, x\right) \equiv-\sum_{n} f_{1 n}\left(\hat{V}, \kappa^{2}\right)$ $e^{i n \alpha} \frac{\left|n \omega_{d \hat{V}, n}\right|_{K^{2}=0}{ }^{2}}{I_{0}} \partial_{\hat{V}} f_{M}$.

According to the relationship between the viscosity and the particle flux [21], the general form of the induced toroidal NTV torque density can be written as

$$
T_{\mathrm{NTV}}=-\left\langle R^{2}\right\rangle_{\psi} \tau_{\mathrm{NTV}}^{-1} \rho_{i} V^{\zeta},
$$

$$
\begin{aligned}
\tau_{\mathrm{NTV}}^{-1}= & \left\langle 1 / R^{2}\right\rangle_{\psi} R_{0}^{2} \sum_{j=i, e} \sum_{n} \frac{\sqrt{\epsilon} q^{2} \omega_{t i}^{2}}{2 \sqrt{2} \pi^{3 / 2}}\left|\frac{Z_{i}}{Z_{j}}\right| \\
& \times \lambda_{1, n}\left(1-\omega_{n c, n} / V^{\zeta}\right) .
\end{aligned}
$$

The $n$th Fourier component of Eq. (2) can be written as

$$
I_{1}\left\langle L\left(f_{1 n}\right)\right\rangle_{b}-i I_{2} f_{1 n}+i I_{3}=0,
$$

where $I_{2} \equiv n \omega_{d \alpha} / I_{0}$ and $I_{3} \equiv \frac{\left\langle b_{n}\right\rangle_{b}}{\left.\left\langle b_{n}\right\rangle_{b}\right|_{\kappa^{2}=0}}$.

With the boundary conditions $\left.F \partial_{\kappa^{2}} f_{1 n}\right|_{\kappa^{2}=0}=0$, and $\left.f_{1 n}\right|_{\kappa^{2}=1}=0$ used in [6,7], Eq. (9) can be solved numerically.

The analytic results of the pitch angle integral $I_{\kappa n}$ in different regimes can be obtained from the results in $[6,7,9,18]$. They have been summarized as Eqs. (54), (55), and (60) in [17]. To check the consistency of the NTV from the numerical and the analytic solutions, it is only necessary to compare the $I_{\kappa n}$ in different asymptotic limits.

Figure 1 shows the numerical solutions of Eq. (9) for the thermal particles $x=1$, with the $n=1$ magnetic perturbation $b_{1} \cos (\delta \theta), b_{1}=10^{-3}, \delta \equiv m-n q=0.1$. The left subgraphs are real (blue solid) and imaginary (red dashed) part of the numerical solutions of the perturbed distribution function. The right subgraphs are the corresponding numerical (blue solid line) $I_{1}\left|\partial_{\kappa^{2}} f_{1 n}\right|$. The dashed red line in Figs. 1(b) and 1(d) are that from the analytic solution. Figures 1(a)-1(f) are the results for nonresonant particles with $\omega_{B 0}=0$ and different normalized collisionalities $\quad \nu_{* d 0}=\left.\nu_{* d}\right|_{\nu_{d} / \nu=1}$, here $\quad \nu_{* d} \equiv \frac{\nu_{d} /(2 \epsilon)}{\left|n q \omega_{E}\right|}$. Figures 1(a) and 1(b) are the solutions in the $1 / \nu$ regime [6] with $\nu_{* d 0}=100$. Figure 1(b) shows a good agreement between the numerical result (blue solid) and analytic result (red dashed) in this regime. Figures 1(c) and 1(d) are the solutions in the $\nu-\sqrt{\nu}$ regime [7] with $\nu_{* d 0}=$ 0.001. Figure 1(d) shows a good agreement between the numerical result (blue solid) and analytic result (red dashed) in this regime. The dash-dotted line in Fig. 1(d) indicates the boundary of the collisional boundary layer. It shows that the boundary layer gives the dominant contribution. Figures 1(e) and 1(f) are the solutions in the transition between the $1 / \nu$ and $\nu-\sqrt{\nu}$ regimes with 

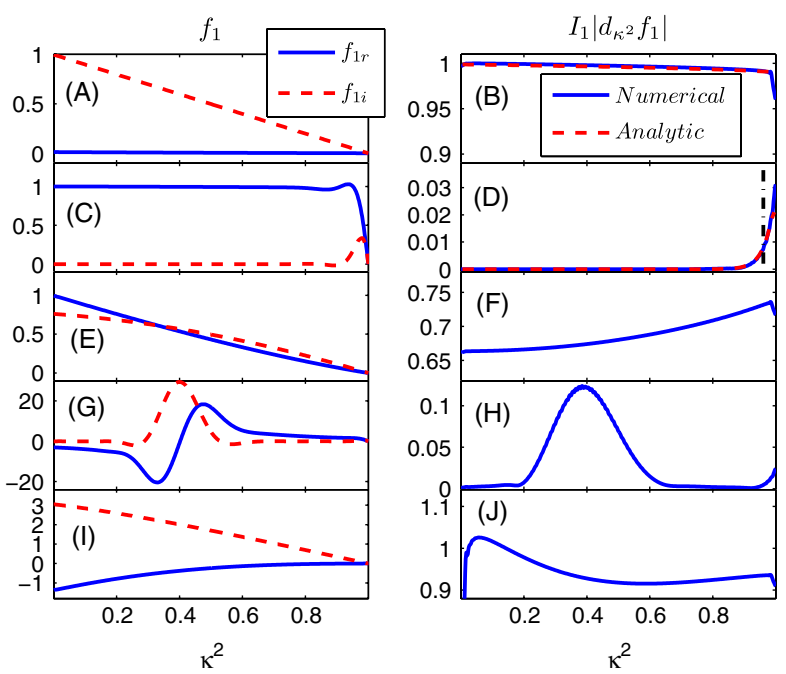

FIG. 1 (color online). The left subgraphs are the real (blue solid) and imaginary (red dashed) part of the numerical solutions of the perturbed distribution function with the following parameters: (a) $\nu_{* d 0}=100, \omega_{B 0}=0$, (c) $\nu_{* d 0}=0.001, \omega_{B 0}=0$, (e) $\nu_{* d 0}=1, \quad \omega_{B 0}=0, \quad(\mathrm{~g}) \quad \nu_{* d 0}=0.001, \quad \omega_{B 0} /\left(q \omega_{E}\right)=$ -1.75 , (i) $\nu_{* d 0}=1, \omega_{B 0} /\left(q \omega_{E}\right)=-1.75$. The right subgraphs are the corresponding numerical (blue solid line) $I_{1}\left|\partial_{\kappa^{2}} f_{1 n}\right|$. The dashed red lines in (b) and (d) are that from the analytic solution. The dash-dotted line in (d) indicates the boundary of the collisional boundary layer.

$\nu_{* d 0}=1$. Figures $1(\mathrm{~g})-1(\mathrm{j})$ are the results for resonant particles with $\left.\omega_{d \alpha}\right|_{\kappa_{0}^{2}=0.4}=0$ by choosing $\omega_{B 0} /\left(q \omega_{E}\right)=$ -1.75 . Figures $1(\mathrm{~g})$ and $1(\mathrm{~h})$ are the solutions in the superbanana plateau regime [9] with $\nu_{* d 0}=0.001$. It is shown in Fig. 1(h) that the resonant part gives the dominant contribution. Figures 1(i) and 1(j) are the solutions in the transition between the $1 / \nu$ and superbanana plateau regimes with $\nu_{* d 0}=1$.

Figure 2 shows the collisionality dependence of the pitch angle integral $I_{k n}$ for the thermal particles with $x=1$ from the numerical solution (blue solid) and analytic solutions by using the Krook operator (red dashed) [18] and smoothly connected formula (black dash-dotted) [17]. Figure 2(a) shows the nonresonant case with $\omega_{B 0}=0$ and Fig. 2(b) shows the resonant case with $\omega_{B 0} /\left(q \omega_{E}\right)=$ -1.75 . The numerical results can well reproduce the results in the $1 / \nu, \nu-\sqrt{\nu}$, and superbanana plateau regimes. The numerical results at the transition regime are different from the results calculated from the smoothly connected formula. There is a bump in the numerical results at the transition between the $1 / \nu$ and superbanana plateau regimes. The difference at this transition shown in this figure can be up to $50 \%$. The solution by using the Krook operator can only give the same results in the superbanana plateau regime. It cannot include the boundary layer effect, and hence, it cannot present the results in the $\sqrt{\nu}$ regime. The results in the $1 / \nu$ regime by using the Krook operator is about twice as large as that by using the pitch angle scattering operator.
The NTV torque has a strong dependence on the collisionality. The NTV torque $T_{\mathrm{NTV}} \propto N^{\alpha} T^{\beta}$, where $\alpha=0$ and $\beta=5 / 2$ in the $1 / \nu$ regime, $\alpha=3 / 2$ and $\beta=1 / 4$ in the $\nu-\sqrt{\nu}$ regime, $\alpha=1$ and $\beta=0$ in the superbanana plateau regime. The radial electric field is related to the plasma rotation via the radial force balance. Therefore, the NTV torque has a complex dependence on the plasma rotation and radial electric field.

Figure 3 shows the dependence of the ion NTV torque $\left(\lg \left[-T_{\mathrm{NTV}}\right]\right)$ on the toroidal plasma rotation and collisionality. The typical plasma parameters in the core region of JET are used in this calculation: $B_{0}=2 T, R_{0}=3 \mathrm{~m}$, $r=0.2 m, \quad L_{T_{i}} \equiv\left|T_{i} / \frac{d T_{i}}{d r}\right|=1 \mathrm{~m}$, the $n=1$ magnetic perturbation $b_{1} \cos (\delta \theta), b_{1}=2 \times 10^{-3}, \delta=0.1$. The plasma rotation is scanned in the range $V^{\zeta}=\omega_{\phi}=$ $(1-100) \mathrm{krad} / \mathrm{s}$. The collisionality is scanned by changing the plasma density in the range $(0.1-1) \times 10^{20} \mathrm{~m}^{-3}$ and keeping the ion pressure as a constant value $P_{i}=$ $3 \mathrm{keV} \times 0.6 \times 10^{20} \mathrm{~m}^{-3}$. The neoclassical value of the poloidal rotation, $V^{\theta} \approx k_{n c} \omega_{* T i}, k_{n c}=1.17$ for the case without momentum source or sink [19,22], is used for radial electric field calculation.

There are obviously three regimes shown in Fig. 3. The red dashed line indicates the location of $\nu_{* d 0}=1$, which is a boundary of the $1 / \nu$ regime (lower right of the line) and the general $\nu-\sqrt{\nu}$ regime (upper left of the line). In the general $\nu-\sqrt{\nu}$ regime, the effect of the resonant particles is important for $x_{\min }$ close to 1 , which gives the superbanana plateau regime (lower left). The boundary between the superbanana plateau regime and the $\sqrt{\nu}$ regime (upper right) is about $x_{\min } \equiv\left|q \omega_{E} / \omega_{B 0}\right| \approx 5$, not $x_{\min }=1$. This means that particles with slightly higher energy than thermal particles can already contribute a torque dominant to the $\sqrt{\nu}$ boundary layer effects.

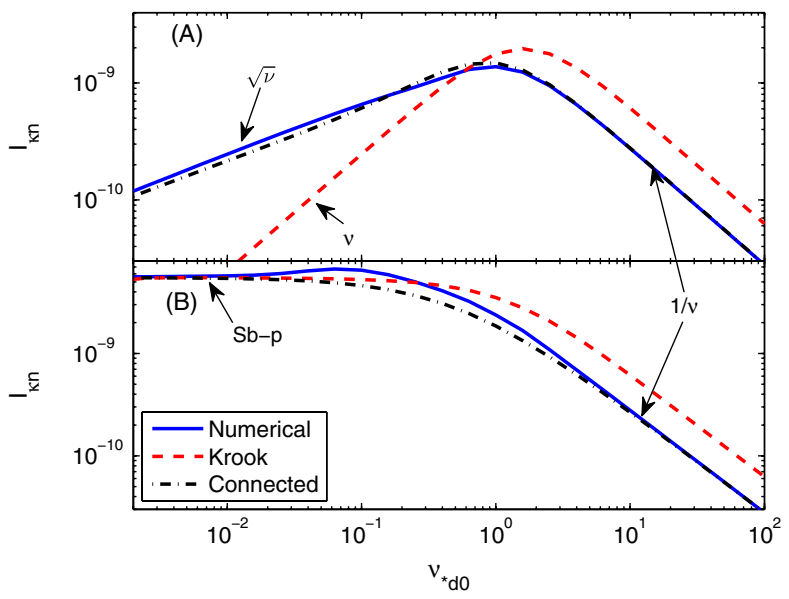

FIG. 2 (color online). The collisionality dependence of the pitch angle integral $I_{\kappa n}$ from the numerical solution (blue solid) and analytic solutions with Krook operator (red dashed) and smoothly connected formula (black dash-dotted) for (a) nonresonant case, $\omega_{B 0}=0$, and (b) resonant case, $\omega_{B 0} /\left(q \omega_{E}\right)=-1.75$. 


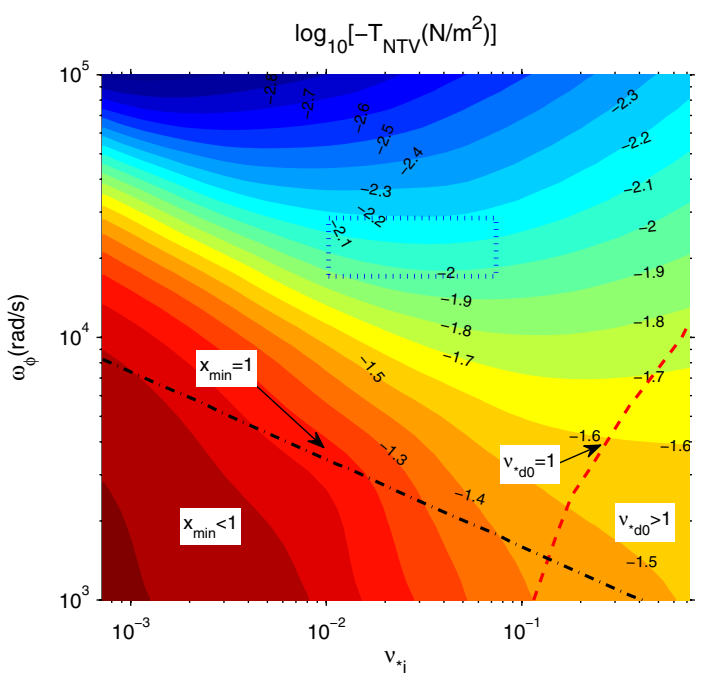

FIG. 3 (color online). The dependence of the ion NTV torque $\left(\lg \left[-T_{\mathrm{NTV}}\right]\right)$ on the plasma rotation and collisionality. The red dashed line indicates $\nu_{* d 0}=1$ and the black dash-dotted line indicates $x_{\min }=1$. The area inside the dotted rectangle is the typical JET regime.

It is shown in Fig. 3 that the torque increases with the decreasing collisionality for the low collisionality case $\left(\nu_{* i}<0.1\right)$ with a plasma rotation in the range $\omega_{\phi} \approx$ 20-30 krad/s, which is the typical plasma rotation with NAMP on JET. It is close to the transition between the $\nu-$ $\sqrt{\nu}$ and superbanana plateau regimes. The collisionality dependence in this regime is mainly contributed from the resonant particles. The enhancement of the NTV torque caused by the resonant particles makes the NTV torque to be more important for the lower collisionality and lower rotation cases, which are the ITER relevant ones.

The resonant particles can give an even stronger effect for negative radial electric field than for positive one. Therefore, the NTV torque will also be very large, if we further reduce the rotation until it goes to the countercurrent direction but it is still smaller than the offset rotation speed. This strong NTV torque will accelerate the plasma in the countercurrent direction, which has been observed on DIII-D [13].

In summary, a general form of the NTV torque in the collisionless regimes in tokamaks, Eq. (7), is obtained in this Letter. The perturbed distribution function is solved numerically from the bounce-averaged drift kinetic equation. In different asymptotic limits of the collisionless regimes, the numerical solutions are in good agreement with the analytic results. The analytic results are different from the numerical results in the transient regimes. This numerical method can be applied for modeling the plasma momentum dissipation caused by NTV in these collisionless regimes and their transitions in tokamaks without additional approximations. The NTV torque has a nonlinear dependence on the radial electrical field and the plasma rotation. The effect of the resonant particles makes the NTV torque more important for the lower collisionality and lower rotation cases, which are the ITER relevant conditions. In addition, the NTV torque due to the resonant particle effects has a strong acceleration effect to the countercurrent direction for the low rotation case.

This work, supported by the European Communities under the contract of Association between EURATOM and FZJ, was carried out within the framework of the European Fusion Development Agreement. The views and opinions expressed herein do not necessarily reflect those of the European Commission.

*y.sun@fz-juelich.de

[1] T. Evans et al., Phys. Rev. Lett. 92, 235003 (2004).

[2] Y. Liang et al., Phys. Rev. Lett. 98, 265004 (2007).

[3] E. J. Strait et al., Phys. Plasmas 11, 2505 (2004).

[4] A. A. Galeev et al., Phys. Rev. Lett. 22, 511 (1969).

[5] D. A. Spong, Phys. Plasmas 12, 056114 (2005).

[6] K. C. Shaing, Phys. Plasmas 10, 1443 (2003).

[7] K. C. Shaing et al., Phys. Plasmas 15, 082506 (2008).

[8] K. C. Shaing, S. A. Sabbagh, and M. S. Chu, Plasma Phys. Controlled Fusion 51, 035004 (2009).

[9] K. C. Shaing, S. A. Sabbagh, and M. S. Chu, Plasma Phys. Controlled Fusion 51, 035009 (2009).

[10] K. C. Shaing, S. A. Sabbagh, and M. S. Chu, Plasma Phys. Controlled Fusion 51, 055003 (2009).

[11] K. C. Shaing, M. S. Chu, and S. A. Sabbagh, Plasma Phys. Controlled Fusion 51, 075015 (2009).

[12] W. Zhu et al., Phys. Rev. Lett. 96, 225002 (2006).

[13] A. M. Garofalo et al., Phys. Rev. Lett. 101, 195005 (2008).

[14] H. Reimerdes et al., Nucl. Fusion 49, 115001 (2009).

[15] Y. Sun et al., Plasma Phys. Controlled Fusion 52, 105007 (2010).

[16] M. Bécoulet et al., Nucl. Fusion 49, 085011 (2009).

[17] K. C. Shaing, S. A. Sabbagh, and M. S. Chu, Nucl. Fusion 50, 025022 (2010).

[18] J.-K. Park, Allen H. Boozer, and Jonathan E. Menard, Phys. Rev. Lett. 102, 065002 (2009).

[19] F. L. Hindon and R. D. Hazeltine, Rev. Mod. Phys. 48, 239 (1976).

[20] A. J. Cole, C. C. Hegna, and J. D. Callen, Phys. Rev. Lett. 99, 065001 (2007).

[21] K. C. Shaing, Phys. Plasmas 3, 4276 (1996).

[22] S. P. Hirshman and D. J. Sigmar, Nucl. Fusion 21, 1079 (1981). 